\title{
Appropriate Minimal Dose of Gadobutrol for 3D Time- Resolved MRA of the Supra-Aortic Arteries: Comparison with Conventional Single-Phase High-Resolution 3D Contrast- Enhanced MRA
}

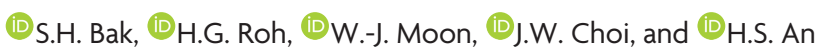

\begin{abstract}
BACKGROUND AND PURPOSE: The development of nephrogenic systemic fibrosis and neural tissue deposition is gadolinium dosedependent. The purpose of this study was to determine the appropriate minimal dose of gadobutrol with time-resolved MRA to assess supra-aortic arterial stenosis with contrast-enhanced MRA as a reference standard.
\end{abstract}

MATERIALS AND METHODS: Four hundred sixty-two consecutive patients underwent both standard-dose contrast-enhanced MRA and low-dose time-resolved MRA and were classified into 3 groups; group A (a constant dose of $1 \mathrm{~mL}$ for time-resolved MRA), group B ( $2 \mathrm{~mL}$ ), or group $C(3 \mathrm{~mL})$. All studies were independently evaluated by 2 radiologists for image quality by using a 5-point scale (from $0=$ failure to $4=$ excellent), grading of arterial stenosis $(0=$ normal, $1=$ mild $[<30 \%], 2=$ moderate $[30 \%-69 \%], 3=$ severe to occlusion $[\geq 70 \%]$, and signal-to-noise ratio.

RESULTS: The image quality of time-resolved MRA was similar to that of contrast-enhanced MRA in groups B and C, but it was inferior to contrast-enhanced MRA in group A. For the grading of arterial stenosis, there was an excellent correlation between contrast-enhanced MRA and time-resolved MRA ( $R=0.957$ for group $\mathrm{A}, R=0.988$ for group $\mathrm{B}, R=0.991$ for group $\mathrm{C}$ ). The SNR of time-resolved MRA tended to be lower than that of contrast-enhanced MRA in groups A and B. However, SNR was higher for time-resolved MRA compared with contrast-enhanced MRA in group C.

CONCLUSIONS: Low-dose time-resolved MRA is feasible in the evaluation of supra-aortic stenosis and could be used as an alternative to contrast-enhanced MRA for a diagnostic technique in high-risk populations.

ABBREVIATIONS: $C C A=$ common carotid artery; $C E-M R A=$ contrast-enhanced MRA; $G B C A=$ gadolinium-based contrast agent; TRICKS = time-resolved imaging of contrast kinetics; TR-MRA = time-resolved MRA

D igital subtraction angiography remains the criterion standard for evaluation of supra-aortic steno-occlusive disease, with excellent spatial and temporal resolution. However, it is a time-consuming and invasive technique and is associated with several risks, including transient ischemic attack, permanent neurologic deficit, iodine contrast nephrotoxicity, and exposure to ionizing radiation. ${ }^{1-3}$ Consequently, DSA has largely been reserved for interventions for extracranial and intracranial stenoocclusive disease or in cases of uncertain findings on noninvasive imaging studies. ${ }^{4}$ Noninvasive angiography techniques such as

Received October 12, 2016; accepted after revision February 13, 2017.

From the Department of Radiology (S.H.B., H.G.R., W.-J.M., J.W.C.), Konkuk University Medical Center, Konkuk University School of Medicine, Seoul, Korea; Department of Radiology (S.H.B.), Kangwon National University Hospital, Chuncheon, Korea; and Department of Radiology (H.S.A.), Armed Forces Yangju Hospital, Yangju, Korea.

Please address correspondence to Hong Gee Roh, MD, PhD, Department of Radiology, Konkuk University Medical Center, Konkuk University School of Medicine, Seoul, Korea, 4-12 Hwayang-dong, Gwangjin-gu, Seoul, 143-729, Korea; e-mail: hgroh@kuh.ac.kr

http://dx.doi.org/10.3174/ajnr.A5176
CTA and MRA are typically used for routine diagnostic procedures. Recently, 3D high-resolution contrast-enhanced MRA (CE-MRA) has become widely used as an excellent alternative imaging technique for the assessment of supra-aortic stenoocclusive disease. $^{5}$

Gadolinium-based contrast agents (GBCAs) were initially thought to be safe in patients with reduced renal function rather than iodine-based contrast agents. ${ }^{6}$ Recently, a positive association between nephrogenic systemic fibrosis and exposure to GBCAs in patients with renal failure has been described. ${ }^{7}$ Several studies showed a relationship between high doses of GBCAs and increased nephrogenic systemic fibrosis risk. ${ }^{8-11}$ Also, in the past 2 years, several studies regarding gadolinium retention in intracranial neuronal tissues have been published. ${ }^{12-14}$ The development of nephrogenic systemic fibrosis with exposure to GBCAs and gadolinium deposition in neuronal tissue is dose-dependent; therefore, caution has been advised when administering GBCAs. ${ }^{13,15}$ There is increasing interest in dose-reduction strategies that maintain diagnostic image quality. ${ }^{16}$ Time-resolved MRA (TR-MRA) is used clinically to offer combined anatomic 


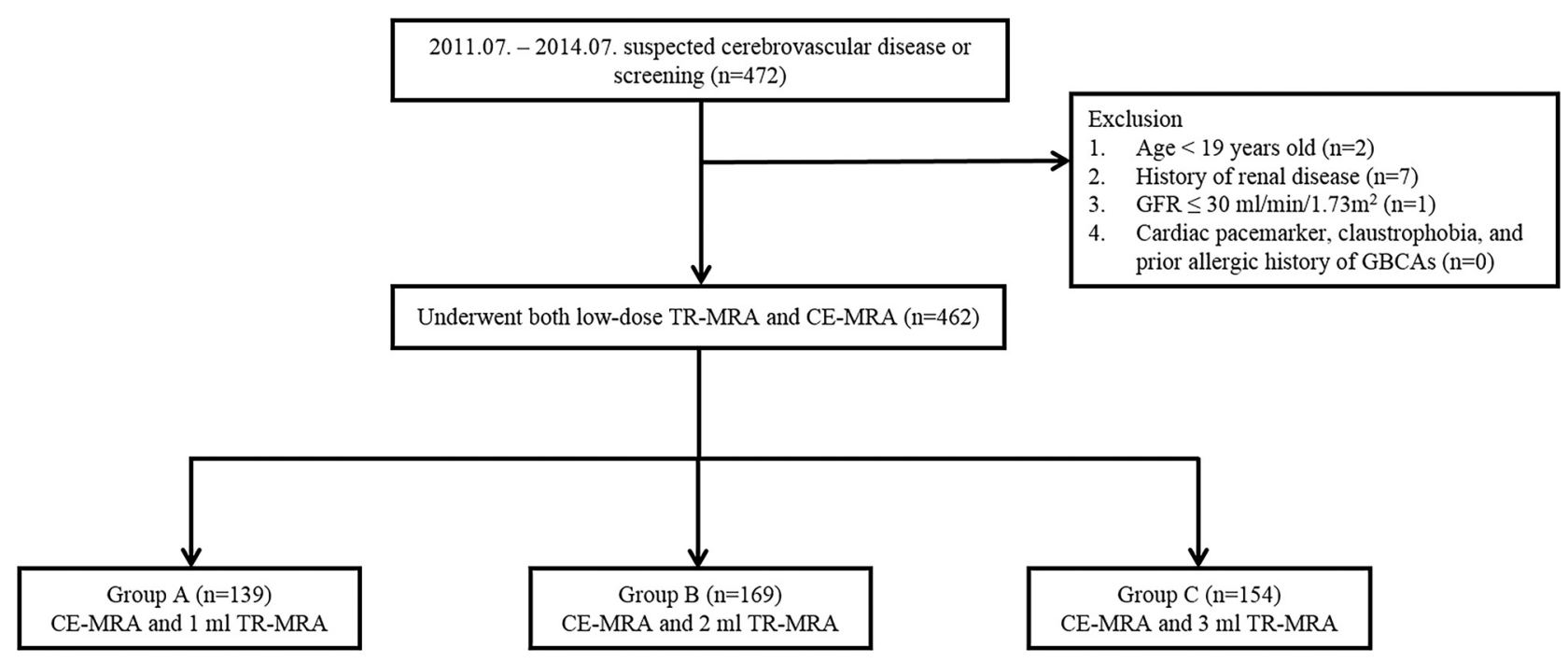

FIG 1. Flow chart of the study population.

and hemodynamic information of the supra-aortic vessels, and another advantage of TR-MRA is the requirement for low-dose GBCAs. ${ }^{2,3,16,17}$ Several studies have demonstrated that TR-MRA with low-dose GBCAs yields comprehensive anatomic and functional information with high sensitivity and negative predictive values. $^{2,3,17-20}$

The purpose of this study was to determine the appropriate minimal dose for TR-MRA to assess supra-aortic arterial stenosis with CE-MRA as a reference standard.

\section{MATERIALS AND METHODS Study Population}

This retrospective study was approved by the institutional review board of Konkuk University Hospital, and informed consent was waived. Between July 2011 and July 2014, 462 consecutive patients underwent both standard-dose CE-MRA and low-dose TR-MRA. TR-MRA was performed with reducing GBCA doses at intervals of 1 year $(3,2$, and $1 \mathrm{~mL}$ of $1 \mathrm{mmol} / \mathrm{mL}$ of GBCA in each year). Clinical indications for MRA included suspected stroke $(n=$ $231)$, vertigo/dizziness $(n=119)$, headache $(n=49)$, seizure $(n=$ $10)$, general examination $(n=25)$, visual disturbance $(n=11)$, tremor $(n=7)$, carotid stenosis $(n=4)$, aneurysm $(n=1)$, hearing difficulty $(n=1)$, tinnitus $(n=2)$, and metabolic disease $(n=2)$. All patients were classified into 3 groups by contrast dose. Group A $(n=139)$, group B $(n=169)$, and group C $(n=154)$ received a constant dose of 1,2 , and $3 \mathrm{~mL}$ of GBCA for TR-MRA, respectively (Fig 1). Exclusion criteria included standard contraindications to MR imaging (eg, cardiac pacemaker, claustrophobia, and prior allergic history of GBCAs), patients younger than 19 years of age, history of renal disease, and a glomerular filtration rate of $<30 \mathrm{~mL} / \mathrm{min} / 1.73 \mathrm{~m}^{2}$.

\section{Image Acquisition}

All examinations were performed on a 3T MR imaging system (Signa HDx; GE Healthcare, Milwaukee, Wisconsin) with a 16channel HNS coil (GE Healthcare). All examinations were performed by experienced technicians and were supervised by experienced neuroradiologists (H.G.R. and J.W.C.).
The imaging protocol for MRA included supra-aortic TRMRA, followed by CE-MRA. The CE-MRA imaging parameters were as follows: $\mathrm{TR} / \mathrm{TE}=4.5 / 1.5 \mathrm{~ms}$, flip angle $=30^{\circ}$, FOV $=300$ $\mathrm{mm}$, matrix $=448 \times 256$, section thickness $=1.2 \mathrm{~mm}$ interpolated to $0.6 \mathrm{~mm}$, bandwidth $=83.33 \mathrm{kHz}$. For CE-MRA, an automatic power injection (Spectris Solaris EP; MedRad, Indianola, Pennsylvania) of $0.1 \mathrm{mmol} / \mathrm{kg}$ body weight of gadobutrol (Gadovist; Bayer Schering Pharma, Berlin, Germany) was used at a flow rate of $3 \mathrm{~mL} / \mathrm{s}$, followed by a saline chase of $20 \mathrm{~mL}$.

TR-MRA was performed by the repetitive acquisition of coronal 3D imaging slabs extending from the aortic arch to the middle cerebral artery in a caudocranial extent. The TR-MRA method used this study was the commercial technique of time-resolved imaging of contrast kinetics (TRICKS; GE Healthcare) MRA. The TRICKS MRA imaging parameters were as follows: TR/TE $=3.1$ / $1.1 \mathrm{~ms}$, flip angle $=20^{\circ}$, FOV $=320 \mathrm{~mm}$, matrix $=320 \times 192$, section thickness $=2.8 \mathrm{~mm}$ interpolated to $0.7 \mathrm{~mm}$, bandwidth $=$ $83.33 \mathrm{kHz}$, and temporal resolution $=2.5$ seconds. A constant dose of $1 \mathrm{~mL}$ (group A), $2 \mathrm{~mL}$ (group B), or $3 \mathrm{~mL}$ (group C) of gadobutrol was injected at a flow rate of $3 \mathrm{~mL} / \mathrm{s}$, followed by a saline flush of $20 \mathrm{~mL}$. TR-MRA was initiated 12 seconds after the injection of contrast medium.

\section{Image Analysis}

Two radiologists (S.H.B. and H.S.A.) interpreted the postprocessed coronal maximum-intensity-projection images of CEMRA and TR-MRA on a dedicated PACS station. The radiologists were blinded to clinical information and contrast agent dose protocol, but they were not blinded to the MRA techniques. Grading was mainly based on the postprocessing MIP images. When MIP images were unclear, source data were used for analysis. For qualitative analysis, the arterial system was divided into 14 segments: right brachiocephalic artery, right and left subclavian arteries, right and left common carotid arteries (CCAs), right and left extracranial internal carotid arteries, right and left intracranial ICAs, right and left vertebral artery orifices, right and left vertebral arteries, and the basilar artery. 
Both CE-MRA and TR-MRA datasets were assessed for image quality. Each arterial segment was graded for image quality with a 5-point scale: 0 = failure of segmental visualization, $1=$ poorly visualized with severe blurring and/or artifacts, $2=$ moderate visualization with moderate blurring and/or artifacts, $3=$ good segmental visualization with minimal blurring and/or artifacts, and $4=$ excellent segmental depiction with sharp margins and the absence of blurring and artifacts. ${ }^{5,20}$ Grade 3 or 4 was considered diagnostic visualization with clear separation of the lumen from the background, whereas grades 0-2 were classified as nondiagnostic visualization. Each reader assessed the entire vessel from its origin to the termination for qualitative analysis. ${ }^{5}$

Contaminating venous enhancement was assessed by using a 4 -point scale: $0=$ no venous contamination, $1=$ minimal with no influence on image interpretation, 2 = moderate limiting diagnostic confidence, and $3=$ severe venous contamination with markedly limited diagnostic interpretation.

Stenosis was rated by using a 4 -point scale with $0=$ no stenosis, $1=$ mild stenosis ( $<30 \%$ luminal reduction), $2=$ moderate stenosis $(30 \%-69 \%)$, and $3=$ severe stenosis to occlusion $(\geq 70 \%) .{ }^{21}$ When $\geq 2$ stenoses were detected, the most severe stenosis was selected for grading.

The signal-to-noise ratio was measured by 1 radiologist (S.H.B.). To evaluate the SNR, the ROI for the arterial segment was placed on each of 12 segmental arteries (right brachiocephalic artery, right and left subclavian arteries, right and left CCAs, right and left extracranial ICAs, right and left intracranial ICAs, right and left vertebral arteries, and the basilar artery) and the background ROI was applied at the right MCA level. The SNR was

Table 1: Demographic characteristics ${ }^{\mathrm{a}}$

\begin{tabular}{|c|c|c|c|c|}
\hline & $\begin{array}{c}\text { Group A } \\
(1 \mathrm{~mL}) \\
(n=139)\end{array}$ & $\begin{array}{c}\text { Group B } \\
(2 \mathrm{~mL}) \\
(n=169)\end{array}$ & $\begin{array}{c}\text { Group C } \\
(3 \mathrm{~mL}) \\
(n=154)\end{array}$ & $\begin{array}{c}P \\
\text { Value }\end{array}$ \\
\hline Age (yr) & $60.3 \pm 13.6$ & $62.2 \pm 13.4$ & $63.2 \pm 14.5$ & .184 \\
\hline Male/female & $80: 59$ & $94: 75$ & $83: 71$ & .821 \\
\hline Body weight (kg) & $63.4 \pm 11.6$ & $63.9 \pm 10.3$ & $62.4 \pm 10.1$ & .498 \\
\hline $\begin{array}{l}\text { Contrast dose of } \\
\text { CE-MRA }(\mathrm{mL})\end{array}$ & $6.3 \pm 1.2$ & $6.4 \pm 1.0$ & $6.2 \pm 1.0$ & .498 \\
\hline $\begin{array}{l}\text { Time between CE-MRA } \\
\text { and TR-MRA (sec) }\end{array}$ & $224 \pm 21$ & $251 \pm 42$ & $233 \pm 36$ & .532 \\
\hline
\end{tabular}

calculated as the signal intensity of the segmental artery divided by the SD of the background noise.

\section{Statistical Analysis}

The Wilcoxon signed rank test was used to evaluate statistical differences between image quality ratings on CE-MRA and TRMRA. The Kruskal-Wallis test was used to test for statistical differences in image quality scores assigned to the TR-MRA among the 3 groups. Agreement between CE-MRA and TR-MRA for grading of stenoses was assessed by the Spearman correlation coefficients $(R)$. A paired $t$ test was performed for assessment of the significant differences in SNR between CE-MRA and TR-MRA. For TR-MRA, 1-way ANOVA was used to assess the SNR among the 3 groups. Interobserver agreement for the image quality and grading of arterial stenosis between 2 readers was calculated by the $\kappa$ coefficient $(\kappa=0$, poor agreement; $\kappa=0.01-0.2$, slight agreement; $\kappa=0.21-0.40$, fair agreement; $\kappa=0.41-0.60$, moderate agreement; $\kappa=0.61-0.80$, good agreement; $\kappa=0.80-1.0$, excellent agreement). $P<.05$ was considered statistically significant. All analyses were performed by using SPSS for Windows, Version 22.0 (IBM, Armonk, New York).

\section{RESULTS}

All patients completed the examination without complications. There were no adverse effects related to the GBCAs. The characteristics of patients are shown in Table 1. Mean body weight was $63.4 \mathrm{~kg}$ in group A (80 men, 59 women; mean age, $60.3 \pm 13.6$ years), $63.9 \mathrm{~kg}$ in group B (94 men, 75 women; mean age, $62.2 \pm$ 13.4 years), and $62.4 \mathrm{~kg}$ in group C ( 83 men, 71 women; mean age, $63.2 \pm 14.5$ years $)$. Clinical characteristics were not significantly different among 3 groups.

\section{Evaluation of Arterial Visualization}

For group A, the mean image-quality scores in 1946 arterial segments were 3.75 (range, 1-4) for CE-MRA and 3.45 (range, 1-4) for TR-MRA. The $\kappa$ coefficient revealed excellent interobserver agreement in the scoring of image quality for both CE-MRA $(\kappa=$ $0.985)$ and TR-MRA $(\kappa=0.972)$. The distribution of scores of image quality is shown in Table 2. Except for right/left intracranial ICAs, arterial delineation scores of CE-MRA in all arterial seg-

Table 2: Comparison of image quality between 2 MRA techniques in the 3 groups

\begin{tabular}{|c|c|c|c|c|c|c|c|c|c|c|c|c|c|c|c|}
\hline \multirow[b]{3}{*}{ Arterial Segment } & \multicolumn{5}{|c|}{ Group A } & \multicolumn{5}{|c|}{ Group B } & \multicolumn{5}{|c|}{ Group C } \\
\hline & \multicolumn{2}{|c|}{ CE-MRA } & \multicolumn{2}{|c|}{ TR-MRA } & \multirow[b]{2}{*}{$P$} & \multicolumn{2}{|c|}{ CE-MRA } & \multicolumn{2}{|c|}{ TR-MRA } & \multirow[b]{2}{*}{$P$} & \multicolumn{2}{|c|}{ CE-MRA } & \multicolumn{2}{|c|}{ TR-MRA } & \multirow[b]{2}{*}{$P$} \\
\hline & Mean & Range & Mean & Range & & Mean & Range & Mean & Range & & Mean & Range & Mean & Range & \\
\hline Right brachiocephalic trunk & 3.96 & $3-4$ & 3.58 & $2-4$ & .000 & 3.97 & $3-4$ & 3.91 & $3-4$ & .001 & 3.97 & $3-4$ & 3.93 & $3-4$ & .034 \\
\hline Right subclavian artery & 3.93 & $2-4$ & 3.58 & $1-4$ & .000 & 3.92 & $1-4$ & 3.92 & $1-4$ & .655 & 3.93 & $2-4$ & 3.94 & $3-4$ & .414 \\
\hline Left subclavian artery & 3.77 & $1-4$ & 3.27 & $1-4$ & .000 & 3.90 & $2-4$ & 3.85 & $2-4$ & .050 & 3.94 & $3-4$ & 3.90 & $3-4$ & .058 \\
\hline Right CCA & 3.91 & $2-4$ & 3.53 & $1-4$ & .000 & 3.95 & $3-4$ & 3.91 & $3-4$ & .052 & 3.93 & $3-4$ & 3.92 & $3-4$ & .655 \\
\hline Right extracranial ICA & 3.99 & $3-4$ & 3.54 & $3-4$ & .000 & 3.99 & $3-4$ & 3.96 & $3-4$ & .059 & 3.99 & $3-4$ & 4.00 & $4-4$ & .317 \\
\hline Right intracranial ICA & 3.39 & $1-4$ & 3.67 & $2-4$ & .000 & 3.45 & $2-4$ & 3.88 & $3-4$ & .000 & 3.56 & $2-4$ & 3.91 & $2-4$ & .000 \\
\hline Left CCA & 3.83 & $2-4$ & 3.22 & $1-4$ & .000 & 3.92 & $3-4$ & 3.79 & $3-4$ & .000 & 3.94 & $3-4$ & 3.92 & $3-4$ & .180 \\
\hline Left extracranial ICA & 3.99 & $3-4$ & 3.61 & $3-4$ & .000 & 3.98 & $3-4$ & 3.96 & $3-4$ & .180 & 3.99 & $3-4$ & 4.00 & $4-4$ & .157 \\
\hline Left intracranial ICA & 3.38 & $1-4$ & 3.63 & $2-4$ & .000 & 3.54 & $2-4$ & 3.89 & $3-4$ & .000 & 3.62 & $2-4$ & 3.90 & $3-4$ & .000 \\
\hline Right vertebral artery orifice & 3.79 & $1-4$ & 3.33 & $1-4$ & .000 & 3.74 & $2-4$ & 3.75 & $2-4$ & .317 & 3.77 & $3-4$ & 3.78 & $3-4$ & .705 \\
\hline Right vertebral artery & 3.51 & $1-4$ & 3.22 & $1-4$ & .000 & 3.62 & $2-4$ & 3.81 & $2-4$ & .000 & 3.62 & $2-4$ & 3.90 & $3-4$ & .000 \\
\hline Left vertebral artery orifice & 3.72 & $3-4$ & 3.19 & $1-4$ & .000 & 3.76 & $3-4$ & 3.75 & $2-4$ & .405 & 3.77 & $3-4$ & 3.77 & $3-4$ & .808 \\
\hline Left vertebral artery & 3.55 & $1-4$ & 3.20 & $2-4$ & .000 & 3.68 & $2-4$ & 3.79 & $2-4$ & .006 & 3.72 & $2-4$ & 3.90 & $2-4$ & .000 \\
\hline Basilar artery & 3.85 & $1-4$ & 3.69 & $2-4$ & .000 & 3.88 & $2-4$ & 3.88 & $2-4$ & 1.000 & 3.91 & $1-4$ & 3.94 & $1-4$ & .157 \\
\hline
\end{tabular}




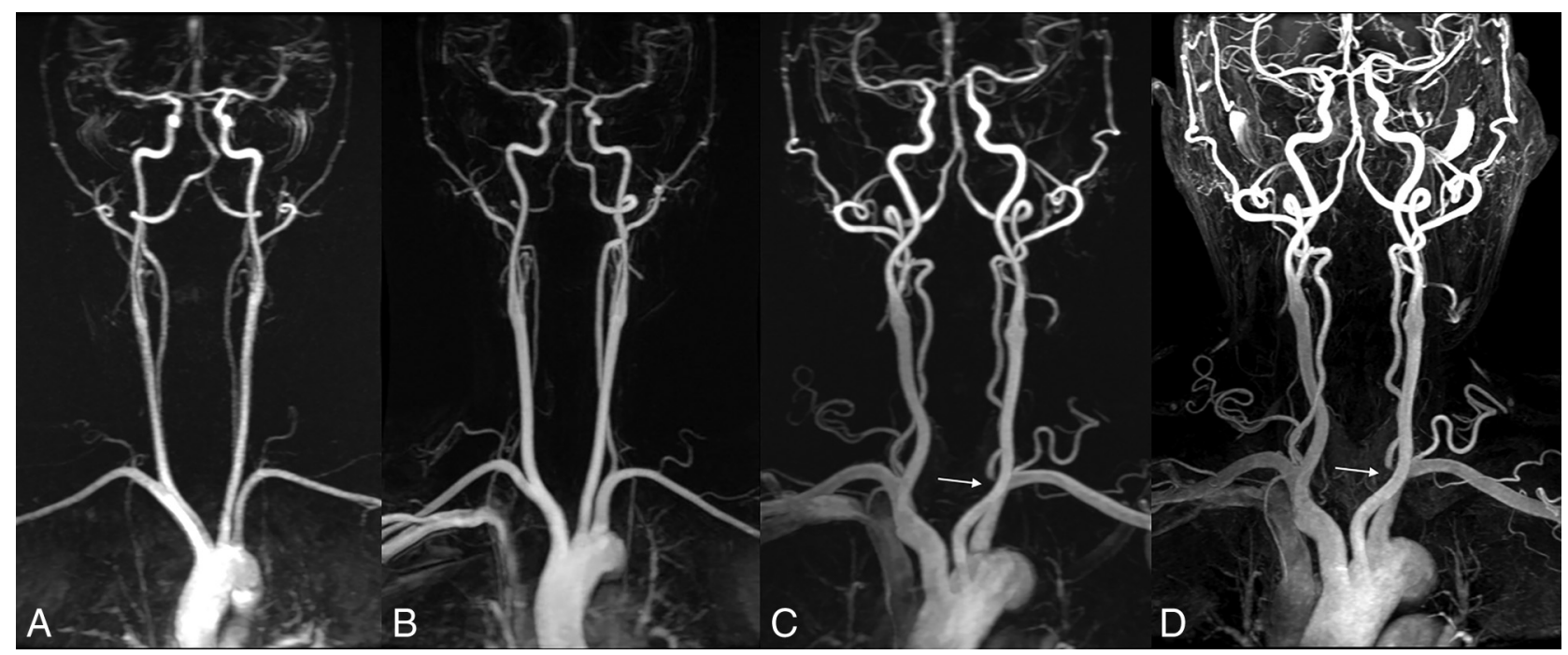

FIG 2. A, TR-MRA with $1 \mathrm{~mL}$ of gadobutrol with subtracted coronal MIP images shows good segmental visualization with minimal blurring or undulation of both CCAs, the extracranial ICA, and the vertebral artery. TR-MRA with $2(B)$ and $3 \mathrm{~mL}(C)$ of gadobutrol with subtraction coronal MIP images shows clear arterial visualization. $D, C E-M R A$ shows mild venous contamination with no influence on the diagnostic interpretation at the intracranial area. TR-MRA with $3 \mathrm{~mL}$ of gadobutrol (C) and CE-MRA (D) show severe stenosis of the left vertebral artery origin (arrow) in a 75 -year-old man with suspected stroke.

Table 3: Distribution of venous contamination at CE-MRA and TR-MRA in the 3 groups $^{\mathrm{a}}$

\begin{tabular}{|c|c|c|c|c|c|c|c|c|c|c|c|c|}
\hline \multirow[b]{2}{*}{ Arterial Segment } & \multicolumn{4}{|c|}{ Group A } & \multicolumn{4}{|c|}{ Group B } & \multicolumn{4}{|c|}{ Group C } \\
\hline & Grade 0 & Grade 1 & Grade 2 & Grade 3 & Grade 0 & Grade 1 & Grade 2 & Grade 3 & Grade 0 & Grade 1 & Grade 2 & Grade 3 \\
\hline Right brachiocephalic trunk & 138/137 & $1 / 1$ & $0 / 1$ & $0 / 0$ & $164 / 165$ & $4 / 4$ & $1 / 0$ & $0 / 0$ & $154 / 154$ & $0 / 0$ & $0 / 0$ & $0 / 0$ \\
\hline Right subclavian artery & $61 / 118$ & $77 / 19$ & $1 / 2$ & $0 / 0$ & $64 / 123$ & $104 / 40$ & $1 / 6$ & $0 / 0$ & $59 / 115$ & $93 / 35$ & $2 / 4$ & $0 / 0$ \\
\hline Left subclavian artery & $128 / 136$ & $11 / 0$ & $0 / 3$ & $0 / 0$ & $164 / 168$ & $5 / 1$ & $0 / 0$ & $0 / 0$ & $142 / 147$ & $10 / 6$ & $2 / 1$ & $0 / 0$ \\
\hline Right CCA & $110 / 134$ & $29 / 4$ & $0 / 1$ & $0 / 0$ & $146 / 156$ & $23 / 12$ & $0 / 1$ & $0 / 0$ & $112 / 139$ & $41 / 14$ & $1 / 1$ & $0 / 0$ \\
\hline Right extracranial ICA & $25 / 101$ & $114 / 38$ & $0 / 0$ & $0 / 0$ & $21 / 119$ & $148 / 50$ & $0 / 0$ & $0 / 0$ & $5 / 111$ & $148 / 43$ & $1 / 0$ & $0 / 0$ \\
\hline Right intracranial ICA & $24 / 108$ & $109 / 31$ & $6 / 0$ & $0 / 0$ & $24 / 120$ & $138 / 49$ & $6 / 0$ & $1 / 0$ & $30 / 114$ & $120 / 39$ & $4 / 1$ & $0 / 0$ \\
\hline Left CCA & $125 / 133$ & $14 / 5$ & $0 / 1$ & $0 / 0$ & $158 / 167$ & $11 / 2$ & $0 / 0$ & $0 / 0$ & $140 / 148$ & $13 / 5$ & $1 / 1$ & $0 / 0$ \\
\hline Left extracranial ICA & $28 / 103$ & $111 / 36$ & $0 / 0$ & $0 / 0$ & 24/111 & $145 / 58$ & $0 / 0$ & $0 / 0$ & $15 / 109$ & $137 / 45$ & $2 / 0$ & $0 / 0$ \\
\hline Left intracranial ICA & $25 / 97$ & $102 / 40$ & $12 / 2$ & $0 / 0$ & 19/115 & $142 / 53$ & $8 / 1$ & $0 / 0$ & $32 / 112$ & $115 / 42$ & $7 / 0$ & $0 / 0$ \\
\hline Right vertebral artery orifice & $122 / 137$ & $17 / 1$ & $0 / 1$ & $0 / 0$ & $151 / 160$ & $17 / 6$ & $1 / 3$ & $0 / 0$ & $132 / 145$ & $22 / 7$ & $0 / 2$ & $0 / 0$ \\
\hline Right vertebral artery & $54 / 112$ & $84 / 27$ & $1 / 0$ & $0 / 0$ & $68 / 139$ & $101 / 29$ & $0 / 1$ & $0 / 0$ & $45 / 126$ & $104 / 27$ & $5 / 1$ & $0 / 0$ \\
\hline Left vertebral artery orifice & $132 / 139$ & $7 / 0$ & $0 / 0$ & $0 / 0$ & $166 / 167$ & $3 / 1$ & $0 / 1$ & $0 / 0$ & 143/151 & $10 / 3$ & $0 / 0$ & $1 / 0$ \\
\hline Left vertebral artery & $51 / 115$ & $86 / 24$ & $2 / 0$ & $0 / 0$ & $63 / 145$ & $105 / 24$ & $1 / 0$ & $0 / 0$ & $51 / 131$ & $101 / 22$ & $2 / 1$ & $0 / 0$ \\
\hline Basilar artery & $29 / 119$ & $103 / 16$ & $6 / 4$ & $1 / 0$ & $51 / 142$ & $105 / 25$ & $13 / 2$ & $0 / 0$ & $36 / 134$ & $111 / 20$ & $7 / 0$ & $0 / 0$ \\
\hline
\end{tabular}

a Data are number of segments for CE-MRA/TR-MRA.

ments was higher than those of TR-MRA, and there was a statistically significant difference between the 2 techniques $(P<.000)$.

For group B, mean image quality scores in 2366 arterial segments were 3.81 (range, 1-4) for CE-MRA and 3.86 (range, 1-4) for TR-MRA. The $\kappa$ coefficient revealed excellent interobserver agreement in the scoring of image quality for both CE-MRA $(\kappa=$ $0.982)$ and TR-MRA $(\kappa=0.971)$. For group C, the mean imagequality scores in a total of 2156 arterial segments were 3.83 (range, 1-4) for CE-MRA and 3.91 (range, 1-4) for TR-MRA. The $\kappa$ coefficient revealed excellent interobserver agreement in the scoring of image quality for both CE-MRA $(\kappa=0.991)$ and TR-MRA $(\kappa=0.969)$. For groups B and C, most of the arterial segments had no statistically significant difference in scores of image quality between the 2 techniques $(P>.05)$. However, arterial delineation scores of TR-MRA in the right/left intracranial ICAs and right/left vertebral arteries were statistically significantly higher than those of CE-MRA in groups B and C (Table 2).

With regard to TR-MRA, age, sex, and body weight-adjusted arterial delineation scores among the 3 groups were statistically different $(P<.000)$. As the dose increased, the arterial delineation scores of TR-MRA showed a tendency to improve $($ mean $=3.43$ for group $\mathrm{A}$, mean $=3.86$ for group $\mathrm{B}$, mean $=3.91$ for group $\mathrm{C}$ ) (Fig 2).

\section{Evaluation of Venous Contamination}

The distribution of venous contamination at CE-MRA and TRMRA in the 3 groups is shown in Table 3. The venous contamination of CE-MRA tended to be higher than that of TR-MRA ( $45.9 \%$ versus $13.2 \%$ in group A, $45.8 \%$ versus 15.6 in group B, $49.2 \%$ versus $14.8 \%$ in group C). On CE-MRA, group A showed $29(1.5 \%)$ segments, group B showed $31(1.3 \%)$, and group C showed $35(1.6 \%)$ segments with moderate-to-severe venous contamination, while group A showed 15 (0.8\%) segments, group B showed 16 (0.7\%) segments, and group C showed $12(0.6 \%)$ segments with moderate-to-severe venous contamination on TR-MRA.

\section{Evaluation of Arterial Stenosis}

The distribution of stenosis at CE-MRA and TR-MRA in the 3 groups is shown in Table 4. Concerning the grading of arterial 
Table 4: Evaluation of stenosis at CE-MRA and TR-MRA in the 3 groups

\begin{tabular}{|c|c|c|c|c|c|c|c|c|c|c|c|c|c|c|c|}
\hline \multirow[b]{2}{*}{ CE-MRA } & \multicolumn{5}{|c|}{ Group A TR-MRA } & \multicolumn{5}{|c|}{ Group B TR-MRA } & \multicolumn{5}{|c|}{ Group C TR-MRA } \\
\hline & 0 & 1 & 2 & 3 & Total & 0 & 1 & 2 & 3 & Total & 0 & 1 & 2 & 3 & Total \\
\hline 0 & 1766 & 2 & 3 & 2 & 1773 & 2149 & 1 & 0 & 0 & 2150 & 1947 & 1 & 0 & 0 & 1948 \\
\hline 1 & 6 & 57 & 4 & 0 & 67 & 0 & 98 & 2 & 0 & 100 & 1 & 102 & 1 & 0 & 104 \\
\hline 2 & 1 & 4 & 69 & 1 & 75 & 3 & 3 & 76 & 0 & 82 & 0 & 4 & 75 & 0 & 79 \\
\hline 3 & 0 & 0 & 0 & 31 & 31 & 0 & 0 & 0 & 34 & 34 & 1 & 0 & 0 & 24 & 25 \\
\hline Total & 1773 & 63 & 76 & 34 & 1946 & 2152 & 102 & 78 & 34 & 2366 & 1949 & 107 & 76 & 24 & 2156 \\
\hline
\end{tabular}

Table 5: Comparison of SNR between 2 MRA techniques in the 3 groups $^{\text {a }}$

\begin{tabular}{|c|c|c|c|c|c|c|c|c|c|}
\hline \multirow[b]{2}{*}{ Arterial Segment } & \multicolumn{3}{|c|}{ Group A } & \multicolumn{3}{|c|}{ Group B } & \multicolumn{3}{|c|}{ Group C } \\
\hline & CE-MRA & TR-MRA & $P$ & CE-MRA & TR-MRA & $P$ & CE-MRA & TR-MRA & $P$ \\
\hline Right brachiocephalic trunk & $211.2 \pm 50.6$ & $144.0 \pm 47.6$ & .000 & $203.0 \pm 45.3$ & $211.2 \pm 61.1$ & .019 & $204.0 \pm 116.0$ & $230.8 \pm 65.9$ & .011 \\
\hline Right subclavian artery & $150.8 \pm 41.0$ & $97.8 \pm 32.0$ & .000 & $144.2 \pm 36.6$ & $154.0 \pm 81.9$ & .099 & $135.2 \pm 37.0$ & $167.4 \pm 110.7$ & .000 \\
\hline Left subclavian artery & $163.3 \pm 35.3$ & $100.9 \pm 29.3$ & .000 & $157.0 \pm 34.0$ & $154.0 \pm 40.4$ & .276 & $152.3 \pm 36.6$ & $174.9 \pm 45.7$ & .000 \\
\hline Right CCA & $172.1 \pm 45.5$ & $103.9 \pm 32.6$ & .000 & $164.8 \pm 42.1$ & $156.4 \pm 48.7$ & .017 & $157.1 \pm 44.9$ & $173.8 \pm 47.3$ & .000 \\
\hline Right extracranial ICA & $190.4 \pm 63.9$ & $107.0 \pm 38.9$ & .000 & $177.4 \pm 53.5$ & $166.4 \pm 57.4$ & .003 & $176.5 \pm 54.5$ & $193.1 \pm 61.3$ & .001 \\
\hline Right intracranial ICA & $280.1 \pm 77.4$ & $136.3 \pm 46.6$ & .000 & $289.9 \pm 172.2$ & $226.3 \pm 74.7$ & .000 & $274.5 \pm 71.3$ & $280.1 \pm 77.2$ & .364 \\
\hline Left CCA & $200.6 \pm 48.8$ & $112.9 \pm 33.3$ & .000 & $189.7 \pm 45.9$ & $172.2 \pm 43.2$ & .000 & $183.6 \pm 50.1$ & $200.2 \pm 52.0$ & .000 \\
\hline Left extracranial ICA & $220.8 \pm 66.0$ & $120.1 \pm 38.5$ & .000 & $204.2 \pm 59.3$ & $184.9 \pm 62.0$ & .000 & $199.8 \pm 61.5$ & $224.1 \pm 70.8$ & .000 \\
\hline Left intracranial ICA & $300.5 \pm 73.2$ & $144.9 \pm 47.1$ & .000 & $295.3 \pm 63.5$ & $240.4 \pm 66.1$ & .000 & $291.1 \pm 70.8$ & $301.7 \pm 82.5$ & .137 \\
\hline Right vertebral artery & $165.3 \pm 52.9$ & $71.8 \pm 27.7$ & .000 & $158.4 \pm 57.3$ & $115.8 \pm 57.9$ & .000 & $158.0 \pm 55.2$ & $136.2 \pm 56.4$ & .000 \\
\hline Left vertebral artery & $169.6 \pm 60.7$ & $73.7 \pm 25.6$ & .000 & $167.4 \pm 53.8$ & $122.1 \pm 49.8$ & .000 & $170.6 \pm 55.3$ & $149.1 \pm 52.2$ & .000 \\
\hline Basilar artery & $223.2 \pm 59.5$ & $80.7 \pm 29.5$ & .000 & $229.9 \pm 55.4$ & $136.1 \pm 62.8$ & .000 & $230.5 \pm 46.9$ & $165.5 \pm 54.9$ & .000 \\
\hline
\end{tabular}

${ }^{a}$ Data are means.

stenosis, there was an excellent correlation between CE-MRA and TR-MRA in the 3 groups $(R=0.957$ for group $\mathrm{A}, R=0.988$ for group $\mathrm{B}, R=0.991$ for group $\mathrm{C}$; all $P<.000)$. For evaluation of arterial stenosis, interobserver agreement for each MRA technique in the 3 groups indicated excellent agreement $(\kappa=$ $0.988-0.998)$.

\section{Vascular SNR}

Table 5 presents a comparison of mean SNR between CE-MRA and TR-MRA in the 3 groups. In groups A and B, the SNR of TR-MRA tended to be significantly lower than that of CE-MRA (204.1 \pm 73.3 for CE-MRA, $107.7 \pm 43.7$ for TR-MRA, $P<.000$ in group A; $199.0 \pm 84.2$ for CE-MRA, $170.8 \pm 71.0$ for TR-MRA, $P<.000$ in group B). The SNR was higher for TR-MRA compared with CE-MRA in group $C$, except for both vertebral arteries and the basilar artery $(194.4 \pm 77.3$ for CE-MRA, $199.7 \pm 82.9$ for TR-MRA, $P=.004)$. With regard to TR-MRA, the measured SNR was highest for group C $(199.7 \pm 82.9)$ followed by group B $(170.8 \pm 71.0)$ and group A $(107.7 \pm 43.7)(P<.000)$.

\section{DISCUSSION}

CE-MRA has emerged as a powerful noninvasive imaging technique for morphologic assessment of steno-occlusive disease and is widely used in clinical practice. ${ }^{5}$ All GBCAs approved for MRA have been considered to have a relatively wide safety margin $(0.1-0.3 \mathrm{mmol} / \mathrm{kg})$ and have been recommended as a substitute for iodine-based contrast agents in patients with reduced renal function in CTA. ${ }^{13,22}$ A significant association between nephrogenic systemic fibrosis and exposure or the dose of GBCAs is well-known; therefore, the use of GBCAs and iodine-based contrast agents is limited in patients with reduced renal function..$^{7-11}$ In the past 2 years, studies have suggested that there is a risk of gadolinium deposition in neural tissue following repeat GBCA administration in patients with normal renal function, and a dose-dependent relationship between gadolinium administration and brain deposition has been described, though the clinical significance of gadolinium retention remains unclear. ${ }^{12,14,15,23}$ A number of attempts have been made to reduce the dose of GBCAs. TR-MRA can provide anatomic or hemodynamic information with a small dose of GBCA. ${ }^{2}$ Therefore, given the findings of nephrogenic systemic fibrosis and gadolinium deposition in the brain, TRMRA has the potential to be an alternative technique. ${ }^{16}$ In our study, low-dose TR-MRA with $1 \mathrm{~mL}$ of GBCA produced suboptimal image quality, whereas the image quality of TR-MRA with 2 or $3 \mathrm{~mL}$ of GBCA was comparable with that of CE-MRA. When using $3 \mathrm{~mL}$, the SNR of TR-MRA was higher than that of CE-MRA. The data demonstrated that TR-MRA with only 1 $\mathrm{mL}$ of GBCA could be useful for detection of supra-aortic arterial stenosis.

In a previous study, TR-MRA with a full dose of GBCA was comparable with DSA for the grading of stenosis. ${ }^{24}$ Lohan et $\mathrm{al}^{20}$ reported that TR-MRA with $3 \mathrm{~mL}$ of Gd-DTPA $(0.5 \mathrm{mmol} /$ $\mathrm{mL}$ ) preserved the overall image quality, whereas image quality with $1.5 \mathrm{~mL}$ of contrast dose was associated with more suboptimal quality. Also, Lee et $\mathrm{al}^{25}$ demonstrated that TR-MRA with an injection of $0.03 \mathrm{mmol} / \mathrm{kg}$ of gadoterate meglumine was feasible and effective in the diagnosis of supra-aortic arterial stenosis. Gadobutrol is a macrocyclic and nonionic agent that has the lowest risk of nephrogenic systemic fibrosis but it can also result in neural tissue deposition. ${ }^{6,13,14,26}$ Similar to previous studies, our study showed that the image quality of TR-MRA with $1 \mathrm{~mL}$ of gadobutrol was inferior to that of CE-MRA, whereas the image quality of TR-MRA with 2 and $3 \mathrm{~mL}$ was not statistically different from that of CE-MRA. Several studies demonstrated that lowdose TR-MRA showed highly concordant results for the evaluation of stenosis. ${ }^{2,3,20}$ Our study showed complementary results. In 

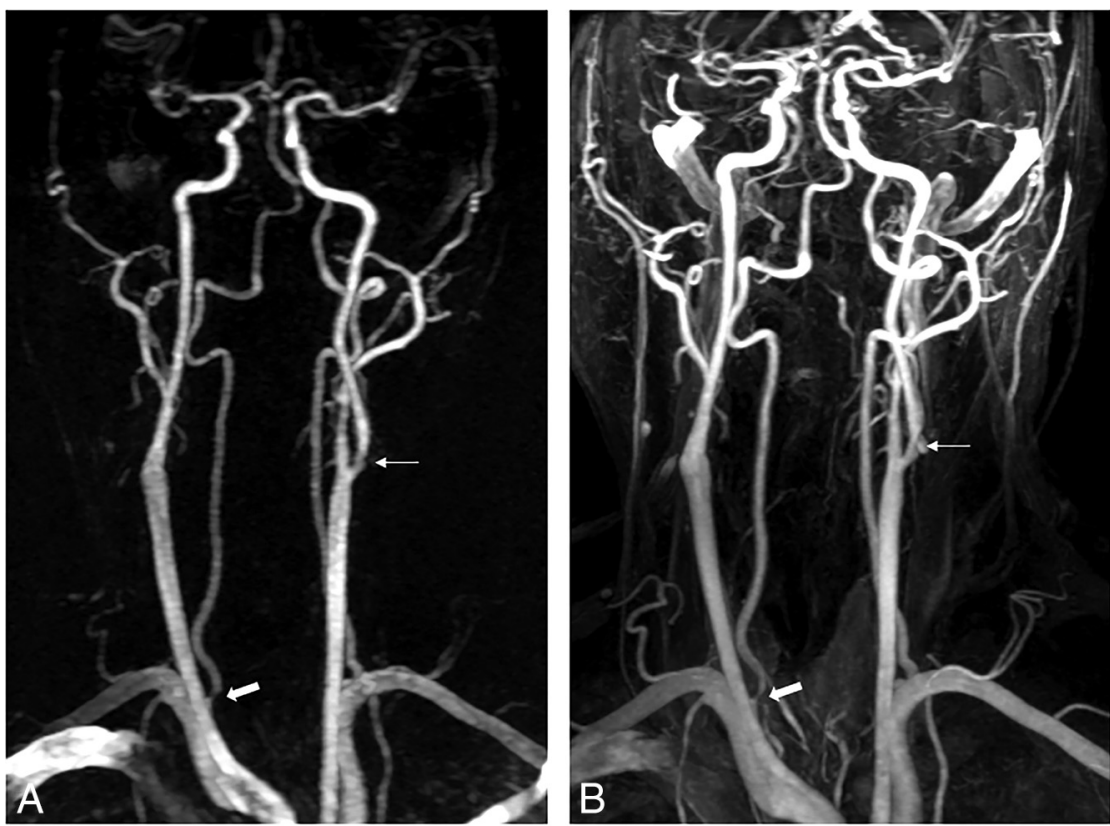

FIG 3. A 52-year-old man with suspected transient ischemic attack. TR-MRA with $1 \mathrm{~mL}$ of gadobutrol $(A)$ and CE-MRA $(B)$ show moderate stenosis at the origin of the right vertebral artery (thick arrow) and mild stenosis of the left proximal extracranial ICA (thin arrow).
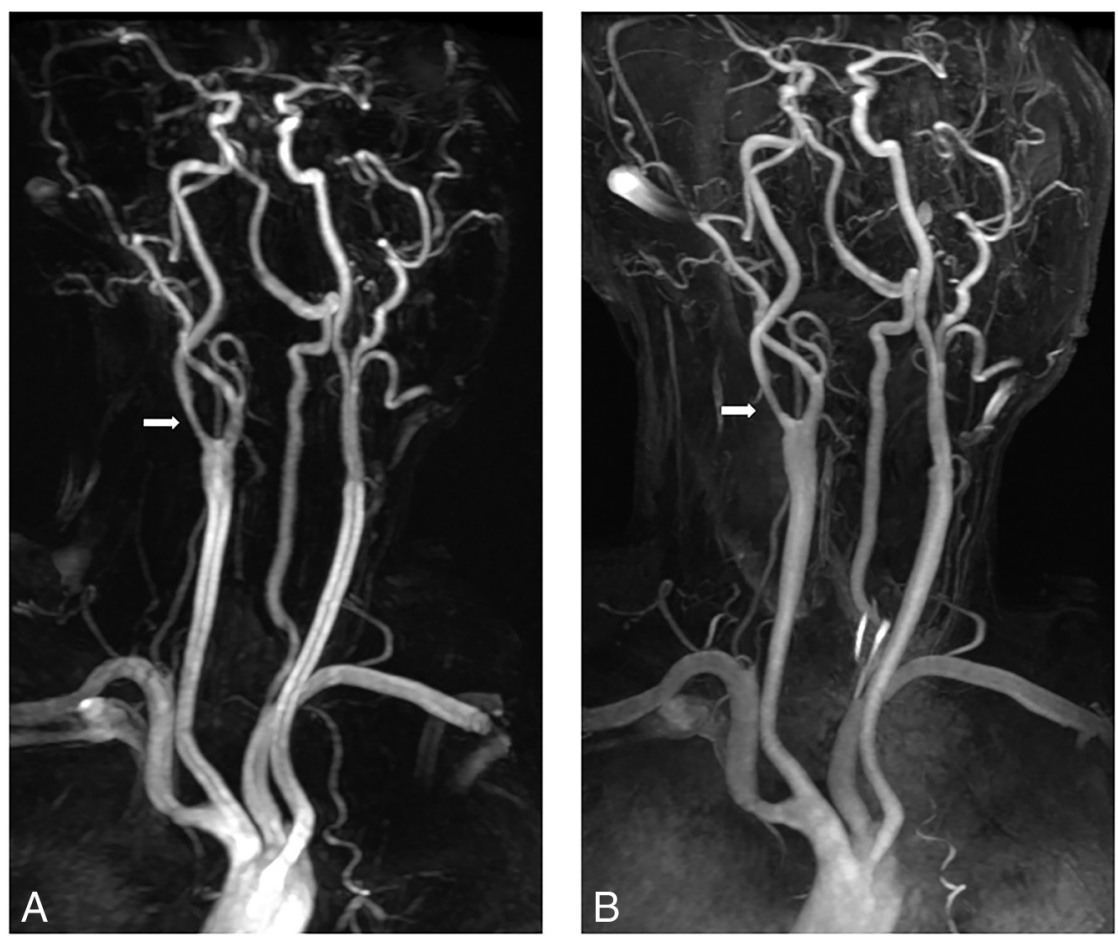

FIG 4. A 74-year-old man with suspected stroke. TR-MRA with $2 \mathrm{~mL}$ of gadobutrol $(A)$ and CE-MRA (B) show moderate stenosis in the proximal extracranial ICA (thick arrow).
On the other hand, TR-MRA enables visualization of the temporal dynamics of blood flow and captures a clear arterial phase with minimal venous contamination. ${ }^{16,30,31}$ Commercial TR-MRA techniques such as TRICKS, time-resolved imaging with stochastic trajectories (TWIST; Siemens, Erlangen, Germany), or 4D TR-MRA with Keyhole (4DTRAK; Philips Healthcare, Best, the Netherlands) should provide a similar level of image quality. ${ }^{32}$ In this study, TRICKS was used. Venous contamination occurred 3 times more often on CEMRA compared with TR-MRA. Venous contamination that influenced image interpretation was mostly seen in both the intracranial ICAs and basilar arteries on CE-MRA (Fig 2). In all 3 groups, image quality of both intracranial ICAs affected by venous enhancement was rated significantly lower for CE-MRA compared with TR-MRA $(P<.000)$.

A high SNR is desirable to accurately depict arterial stenosis. ${ }^{27}$ In our data, the mean SNR of TR-MRA with 1 and $2 \mathrm{~mL}$ of GBCA was inferior to the SNR of CEMRA. TR-MRA effectively eliminated venous contamination, and background signal can be subtracted with the mask images. ${ }^{32}$ Thus, our study shows that the mean SNR was higher for TR-MRA with $3 \mathrm{~mL}$ of GBCA compared with CE-MRA (194.4 \pm 77.3 for CE-MRA, $199.7 \pm$ 82.9 for TR-MRA, $P=.004)$ and an increased SNR of TR-MRA with a dose of $3 \mathrm{~mL}$ led to a similar score of image quality of arterial visualization compared with CE-MRA. Voth et $\mathrm{al}^{33} \mathrm{dem}$ onstrated that full-dose gadolinium MRA results in higher SNR compared with a half dose. With regard to TRMRA, the SNR increased as the dose increased $(199.7 \pm 82.9$ for a dose of $3 \mathrm{~mL}$, $170.8 \pm 71.0$ for a dose of $2 \mathrm{~mL}$, and $107.7 \pm 43.7$ for a dose of $1 \mathrm{~mL}$ ).

The present study has some limitations. First, the study retrospectively compared CE-MRA and TR-MRA, and TR-MRA for grading of stenosis all groups, low-dose TR-MRA had good correlation for the assessment of stenosis compared with CE-MRA $(R=0.957-0.991)$ (Figs 3 and 4).

The key to the success of CE-MRA is the acquisition of the central lines of $k$-space during peak arterial enhancement. ${ }^{27,28}$ However, this is challenging because of rapid arterial-venous transit and the short duration of the arterial phase ( $\sim 10$ seconds) rather than the acquisition time for CE-MRA ( $\sim 30$ seconds). ${ }^{28,29}$ was not compared with DSA or CTA. CE-MRA has been validated as a credible criterion standard for supra-aortic artery imaging. ${ }^{20,25}$ However, CE-MRA was limited in the evaluation of some vessels. For example, CE-MRA has a tendency to overestimate arterial stenosis and has frequently shown pseudostenosis of the vertebral artery origin due to weak spatial resolution, intravoxel dephasing, and motion artifacts. ${ }^{34}$ Validation in a prospective study will be necessary for comparison be- 
tween low-dose TR-MRA and DSA regarding the accuracy in grading of the stenosis. However, the results of our study provide valuable information on the feasibility of low-dose TRMRA with a relatively large number of patients. Second, there were a relatively small number of patients with stenosis because this study was retrospective and most patients underwent MRA for suspected intracranial or extracranial vascular lesions as well as screening. Third, venous contamination of CE-MRA may have been affected by previous TR-MRAs.

\section{CONCLUSIONS}

The image quality and diagnostic agreement for stenotic disease of TR-MRA were not inferior to those of CE-MRA for all 3 doses and appear better for 2- and 3-mL doses compared with a 1-mL dose. Patients with nephropathy and end-stage renal disease become more prevalent recently, and there is an increasing burden of complications associated with GBCA used for diagnosis and management of steno-occlusive disease, ${ }^{16,35}$ though it is unclear whether this finding is clinically significant relative to the risk of the contrast dose. TR-MRA with 2 or $3 \mathrm{~mL}$ of gadobutrol may be used as an alternative to CE-MRA for diagnostic techniques in high-risk populations.

\section{REFERENCES}

1. Krings T, Hans F. New developments in MRA: time-resolved MRA. Neuroradiology 2004;46(suppl 2):s214-22 CrossRef Medline

2. Lee YJ, Laub G, Jung SL, et al. Low-dose 3D time-resolved magnetic resonance angiography (MRA) of the supraaortic arteries: correlation with high spatial resolution 3D contrast-enhanced MRA. $J$ Magn Reson Imaging 2011;33:71-76 CrossRef Medline

3. Lim RP, Shapiro M, Wang EY, et al. 3D time-resolved MR angiography (MRA) of the carotid arteries with time-resolved imaging with stochastic trajectories: comparison with $3 \mathrm{D}$ contrast-enhanced bolus-chase MRA and 3D time-of-flight MRA. AJNR Am J Neuroradiol 2008;29:1847-54 CrossRef Medline

4. Thiex R, Norbash AM, Frerichs KU. The safety of dedicated-team catheter-based diagnostic cerebral angiography in the era of advanced noninvasive imaging. AJNR Am J Neuroradiol 2010;31: 230-34 CrossRef Medline

5. Tomasian A, Salamon N, Lohan DG, et al. Supraaortic arteries: contrast material dose reduction at 3.0-T high-spatial-resolution MR angiography_feasibility study. Radiology 2008;249:980-90 CrossRef Medline

6. Thomsen HS, Morcos SK, Almén T, et al; ESUR Contrast Medium Safety Committee. Nephrogenic systemic fibrosis and gadoliniumbased contrast media: updated ESUR Contrast Medium Safety Committee guidelines. Eur Radiol 2013;23:307-18 CrossRef Medline

7. Zhang B, Liang L, Chen W, et al. An updated study to determine association between gadolinium-based contrast agents and nephrogenic systemic fibrosis. PLoS One 2015;10:e0129720 CrossRef Medline

8. Broome DR, Girguis MS, Baron PW, et al. Gadodiamide-associated nephrogenic systemic fibrosis: why radiologists should be concerned. AJR Am J Roentgenol 2007;188:586-92 CrossRef Medline

9. Collidge TA, Thomson PC, Mark PB, et al. Gadolinium-enhanced MR imaging and nephrogenic systemic fibrosis: retrospective study of a renal replacement therapy cohort. Radiology 2007;245:168-75 CrossRef Medline

10. Kallen AJ, Jhung MA, Cheng S, et al. Gadolinium-containing magnetic resonance imaging contrast and nephrogenic systemic fibrosis: a casecontrol study. Am J Kidney Dis 2008;51:966-75 CrossRef Medline

11. Marckmann P, Skov L, Rossen K, et al. Case-control study of gadodiamide-related nephrogenic systemic fibrosis. Nephrol Dial Transplant 2007;22:3174-78 CrossRef Medline

12. McDonald RJ, McDonald JS, Kallmes DF, et al. Intracranial gadolin- ium deposition after contrast-enhanced MR imaging. Radiology 2015;275:772-82 CrossRef Medline

13. Ramalho J, Semelka RC, Ramalho M, et al. Gadolinium-based contrast agent accumulation and toxicity: an update. AJNR Am J Neuroradiol 2016;37:1192-98 CrossRef Medline

14. Stojanov DA, Aracki-Trenkic A, Vojinovic S, et al. Increasing signal intensity within the dentate nucleus and globus pallidus on unenhanced T1W magnetic resonance images in patients with relapsingremitting multiple sclerosis: correlation with cumulative dose of a macrocyclic gadolinium-based contrast agent, gadobutrol. Eur Radiol 2016;26:807-15 CrossRef Medline

15. Karabulut N. Gadolinium deposition in the brain: another concern regarding gadolinium-based contrast agents. Diagn Interv Radiol 2015;21:269-70 CrossRef Medline

16. Nael K, Moriarty JM, Finn JP. Low dose CE-MRA. Eur J Radiol 2011; 80:2-8 CrossRef Medline

17. Nael K, Michaely HJ, Villablanca P, et al. Time-resolved contrast enhanced magnetic resonance angiography of the head and neck at 3.0 tesla: initial results. Invest Radiol 2006;41:116-24 CrossRef Medline

18. Kramer U, Fenchel M, Laub G, et al. Low-dose, time-resolved, contrast-enhanced 3D MR angiography in the assessment of the abdominal aorta and its major branches at 3 Tesla. Acad Radiol 2010; 17:564-76 CrossRef Medline

19. Krishnam MS, Tomasian A, Lohan DG, et al. Low-dose, timeresolved, contrast-enhanced 3D MR angiography in cardiac and vascular diseases: correlation to high spatial resolution $3 \mathrm{D}$ contrast-enhanced MRA. Clin Radiol 2008;63:744-55 CrossRef Medline

20. Lohan DG, Tomasian A, Saleh RS, et al. Ultra-low-dose, timeresolved contrast-enhanced magnetic resonance angiography of the carotid arteries at 3.0 Tesla. Invest Radiol 2009;44:207-17 CrossRef Medline

21. Ferguson GG, Eliasziw M, Barr HW, et al. The North American Symptomatic Carotid Endarterectomy Trial: surgical results in 1415 patients. Stroke 1999;30:1751-58 CrossRef Medline

22. Bonvento MJ, Moore WH, Button TM, et al. CT angiography with gadolinium-based contrast media. Acad Radiol 2006;13:979-85 CrossRef Medline

23. Stojanov D, Aracki-Trenkic A, Benedeto-Stojanov D. Gadolinium deposition within the dentate nucleus and globus pallidus after repeated administrations of gadolinium-based contrast agents-current status. Neuroradiology 2016;58:433-41 CrossRef Medline

24. Remonda L, Senn P, Barth A, et al. Contrast-enhanced 3D MR angiography of the carotid artery: comparison with conventional digital subtraction angiography. AJNR Am J Neuroradiol 2002;23: 213-19 Medline

25. Lee YJ, Kim BS, Koo JS, et al. Supra-aortic low-dose contrast-enhanced time-resolved magnetic resonance (MR) angiography at 3 T: comparison with time-of-flight $M R$ angiography and high-resolution contrast-enhanced MR angiography. Acta Radiol 2015;56: 673-80 CrossRef Medline

26. Haneder S, Kucharczyk W, Schoenberg SO, et al. Safety of magnetic resonance contrast media: a review with special focus on nephrogenic systemic fibrosis. Top Magn Reson Imaging 2015;24:57-65 CrossRef Medline

27. Carroll TJ, Korosec FR, Petermann GM, et al. Carotid bifurcation: evaluation of time-resolved three-dimensional contrast-enhanced MR angiography. Radiology 2001;220:525-32 CrossRef Medline

28. Du J, Fain SB, Korosec FR, et al. Time-resolved contrastenhanced carotid imaging using undersampled projection reconstruction acquisition. J Magn Reson Imaging 2007;25:1093-99 CrossRef Medline

29. Watts R, Wang Y, Redd B, et al. Recessed elliptical-centric viewordering for contrast-enhanced 3D MR angiography of the carotid arteries. Magn Reson Med 2002;48:419-24 CrossRef Medline

30. Raoult H, Ferré JC, Morandi X, et al. Quality-evaluation scheme for cerebral time-resolved 3D contrast-enhanced MR angiography techniques. AJNR Am J Neuroradiol 2010;31:1480-87 CrossRef Medline 
31. Riederer SJ, Haider CR, Borisch EA, et al. Recent advances in 3D time-resolved contrast-enhanced MR angiography. J Magn Reson Imaging 2015;42:3-22 CrossRef Medline

32. Choi JW, Roh HG, Moon WJ, et al. Time-resolved 3D contrastenhanced MRA on 3.0T: a non-invasive follow-up technique after stent-assisted coil embolization of the intracranial aneurysm. Korean J Radiol 2011;12:662-70 CrossRef Medline

33. Voth M, Michaely HJ, Schwenke C, et al. Contrast-enhanced magnetic resonance angiography (MRA): evaluation of three different contrast agents at two different doses $(0.05$ and $0.1 \mathrm{mmol} / \mathrm{kg})$ in pigs at 1.5 Tesla. Eur Radiol 2011;21:337-44 CrossRef Medline

34. Kim S, Lee S, Choi HS, et al. Pseudostenosis at the origin of the vertebral artery on contrast-enhanced MRA: correlation with aortic motion on dynamic 3D time-resolved contrast-enhanced MRA. $J$ Korean Society of Magnetic Resonance in Medicine 2012;16:236-42

35. Nael K, Krishnam M, Ruehm SG, et al. Time-resolved MR angiography in the evaluation of central thoracic venous occlusive disease. AJR Am J Roentgenol 2009;192:1731-38 CrossRef Medline 